\title{
Nuclear Localisation of Autophagic p62 and Associated Cytoplasmic Beclin-1 and Bcl-2 Expressions in Adenomas and Adenocarcinomas of the Colorectal Regions
}

Ernest Adankwah ${ }^{1,3}$, Kwabena Owusu Danquah ${ }^{1,2^{*}}$, Daniel Gyamfi ${ }^{1}$, Paul Poku Sampene Ossei ${ }^{3}$, Emmanuel Asiamah ${ }^{1}$, Ibrahim A Alsafari ${ }^{4}$ and Tony $^{2}$ Madgwick 5

${ }^{1}$ Department of Medical Laboratory Technology, Faculty of Allied Health Sciences, Kwame Nkrumah University of Science \& Technology, Kumasi, Ghana

${ }^{2}$ Cancer and Infections Research Facility, College of Health Sciences, Kwame Nkrumah University of Science \& Technology, Kumasi, Ghana

${ }^{3}$ Department of Pathology, School of Medical Sciences, College of Health Sciences, Kwame Nkrumah University of Science \& Technology, Kumasi, Ghana

${ }^{4}$ School of Biomolecular Science and Biomedical Science, University College Dublin, Dublin, Republic of Ireland

${ }^{5}$ Faculty of Science and Technology, University of Westminster, London, UK

*Corresponding author: Dr Kwabena Owusu Danquah, Department of Medical Laboratory Technology, Faculty of Allied Health Sciences, Kwame Nkrumah University of Science \& Technology, Private Mail Bag, Kumasi, Ghana, Tel: +233 233224 002; E-mail: kodanquah.chs@knust.edu.gh

Received date: February 21, 2018; Accepted date: March 27, 2018; Published date: April 02, 2018

Copyright: (C2018 Adankwah E, et al. This is an open-access article distributed under the terms of the Creative Commons Attribution License, which permits unrestricted use, distribution, and reproduction in any medium, provided the original author and source are credited.

\begin{abstract}
Background: Autophagy is an important biological process that is involved in cellular homeostasis and survival. Derailment of some cellular autophagic processes affects normal cellular function, resulting in cancers and other disorders. Autophagy related proteins are Beclin-1, a human tumour suppressor, $\mathrm{Bcl}-2$ and $\mathrm{p}^{62}$ have been characterised in most cancers. Particularly, a number of studies have reported a loss of Beclin-1 and up regulation of $\mathrm{Bcl}-2$ and $\mathrm{p}^{62}$ in breast cancers. However, studies regarding the expression of these proteins in colorectal adenomaadenocarcinoma transformation sequence are yet to be described. In this study, we examined the expression patterns of Beclin-1, Bcl-2 and $\mathrm{p}^{62}$ in both colorectal adenomas and adenocarcinomas.
\end{abstract}

Methods: Immunohistochemistry was performed on formalin-fixed paraffin embedded tissue sections from 14 patients with colorectal tumours and the expression patterns were semi-quantitatively evaluated based on the intensity of staining and the percentage of tumour cells stained.

Results: Cytoplasmic Beclin-1 and $\mathrm{p}^{62}$ expression patterns ranged from moderate to high in both tubular adenomas and adenocarcinomas as compared to normal colonic mucosa. Cytoplasmic Bcl-2 expression was moderately expressed in tubular adenomas but negative to low expression was observed in the adenocarcinomas. This study also provided, for the first time, nuclear localization of $\mathrm{p}^{62}$ in only the colorectal adenocarcinomas.

Conclusion: Beclin-1, Bcl-2 and $\mathrm{p}^{62}$ may be up regulated in the transition of colorectal adenomas to adenocarcinomas.

List of Abbreviations: Bcl-2: B-Cell Lymphoma 2; Bcl-xL: B-cell lymphoma Extra Large; BH2/3: Bcl-2 Homology-2/3; HTA: Human Tissue Act; $\mathrm{P}^{62}$ : Nuclearporin 62; ROS: Reactive Oxidative Stress (ROS); TBST: Tris Buffer Saline/ Tween-20; Vps34: Vacuolar Protein Sorting 34.

Keywords: Autophagy; Beclin 1; $\mathrm{p}^{62}$; Bcl-2; Immunohistochemistry; Colorectal; Adenoma; Adenocarcinoma

\section{Background}

Autophagy, an integral part of cellular homeostasis, is essentially crucial in ensuring the survival of cells or organisms through recycling of unwanted proteins and/ or generation of energy for urgent need. To prevent the cellular milieu from clogging up, damaged or "out-ofwork" organelles and misfolded proteins are sequestered in doublemembrane vesicles called autophagosomes and delivered to cytosolic lysosomes for degradation of intracellular contents [1].
Under stress conditions, such as nutrient starvation and oxygen deprivation, autophagy is induced in eukaryotic cells to generate nutrients and energy. Even though the induction of autophagy might ensure cellular survival and homeostasis, excessive degradation of cellular contents could drain the cells of nutrients and energy, resulting in cell death [2].

Autophagy, a tightly controlled complex cellular process, recruits several protein in initiation, vesicle nucleation, fusion and degradation. An important protein, Beclin 1, which complexes with Vps34, is recruited at the site of autophagosome formation through phosphorylation of Ambral [3].

Beclin 1 is mono-allelically deleted in human breast, ovarian and prostate cancers but overexpressed in colorectal and gastric cancers [4]. In addition, mice with knockout Beclin 1 gene have been shown to have high incidence of tumour growth, indicating Beclin 1 may be the tumour suppressor [5]. Li et al., found an increased expression of Beclin 1 in colorectal cancer cells, and this was shown to correlate with 
Citation: Adankwah E, Danquah KO, Gyamfi D, Sampene Ossei PP, Asiamah E, et al. (2018) Nuclear Localisation of Autophagic p62 and Associated Cytoplasmic Beclin-1 and Bcl-2 Expressions in Adenomas and Adenocarcinomas of the Colorectal Regions. J Carcinog Mutagen 9: 314. doi:10.4172/2157-2518.1000314

Page 2 of 6

a longer survival in cancer patients, suggesting that Beclin 1 may play a role in cell death of cancer cells [6].

However, studies by Koukourakis et al., have shown that overexpression of Beclin 1 correlated with tumour aggressiveness and invasion. They proposed that Beclin 1 induction of autophagy does supresses cancer growth but when it is over-expressed it may play a role in tumour aggressiveness and invasion under adverse conditions. Beclin 1 has a $\mathrm{Bcl}-2$ homology-2 (BH3) domain that is able to bind to $\mathrm{BH} 1-\mathrm{BH} 2$ domains of antiapoptotic proteins $\mathrm{Bcl}-2$ and $\mathrm{Bcl}-\mathrm{xL}$ [7].

The binding leads to inhibition of autophagy, suggesting that Beclin 1 may mediate in a cross talk between autophagy and apoptosis [8]. Bcl-2-Beclin 1 complex leads to the inhibition of autophagy-associated cell death [9]. Studies have shown the loss of Bcl-2 in colon cancers [10].

Another equally important protein that is involved in autophagy, $\mathrm{p}^{62}$, serves as a scaffold for cargoes degradation [11]. The $\mathrm{p} 62$ protein is involved in cellular stress, proliferation, survival and inflammation [12]. It has recently been a focus of research due to its dynamic role in cellular homeostasis and carcinogenesis. Its interaction with membrane bound LC3 regulates the degradation of harmful proteins aggregates and organelles via. autophagy [13].

Thus, the inhibition of autophagy results in the accumulation of $\mathrm{p}^{62}$, leading to increase in levels of intracellular reactive oxidative stress (ROS) that facilitates genomic instability, a condition that may favour malignant transformation [14].

Over expression of $\mathrm{p}^{62}$ has been associated with neurodegenerative diseases such as Alzheimer's and Parkinson's disease, as well as many solid tumours $[14,15]$. However, its role in the colorectal adenomaadenocarcinoma transition sequence is yet to be clarified. Although autophagy has been linked with colorectal tumour development, there are limited studies so far done in this area. In addition, a study on the expression patterns of Beclin $1, \mathrm{Bcl}-2$ and $\mathrm{p}^{62}$ in the colorectal adenoma-adenocarcinoma transformation sequence to our knowledge is unavailable.

In this study therefore, the expression patterns of autophagy related proteins, Beclin 1, Bcl-2 and $\mathrm{p}^{62}$ were assessed immunohistochemically in both colorectal adenomas and adenocarcinomas.

\section{Methods}

\section{Samples}

Formalin fixed paraffin embedded (FFPE) of colorectal tissue samples, consisting of 7 tubular adenomas and 7 adenocarcinomas (Duke's Stage B) were retrieved from Queens Hospital, Romford, UK using SNOMED codes with an ethical approval from the Research Ethics sub-Committee of the University of Westminster, in compliance to the Human Tissue Act (HTA). Tissue samples were given designated HTA codes, which were used throughout this study.

\section{Haematoxylin \& Eosin (H\&E) and Immunohistochemical staining}

Haematoxylin and Eosin staining was performed according to standard protocol to confirm the histological representation of the colorectal tumour tissues. Another set of FFPE tissue sections used in $\mathrm{H} \& \mathrm{E}$ staining, were immunostained for Beclin 1, Bcl-2 and p62 as briefly described here. Sectioned tissues were dewaxed, hydrated and blocked with $30 \%$ hydrogen peroxide in methanol for $10 \mathrm{~min}$ to deactivate any endogenous peroxidase activity.

Beclin 1 and $\mathrm{Bcl}-2$ antigens were retrieved using citrate buffer, $\mathrm{pH}$ 6.0, in a pressure cooker for $2 \mathrm{~min}$. Antigen retrieval for p62 was achieved in citrate buffer, $\mathrm{pH} 6.0$, in a microwave. Following antigen retrieval, sections were incubated in $2.5 \%$ normal horse serum for 20 min to block for any non-specific binding sites.

Sections were immunostained with diluted primary antibodies (Table 1) for $60 \mathrm{~min}$ followed by incubation with ImmPRESS regeant (Vector Laboratories Ltd) for $30 \mathrm{~min}$. Colour development was achieved in Vector Impact BAD reagent for Beclin 1, Bcl-2, and p62. The immunostained sections were counterstained with haematoxylin, dehydrated, cleared and mounted. All the washings were performed with Tris buffer saline/ 0.05\% Tween-20 (TBST).

Staining without the primary antibody was performed as a negative control. Staining of the small lymphocytes in the mantle zone of the lymphoid follicle was used as an internal positive control for Bcl-2 and staining at the germinal centre was use as internal positive control for both Beclin 1 and $\mathrm{p}^{62}$.

\begin{tabular}{|l|l|l|l|}
\hline Antibody & Dilution & Clone & Source \\
\hline Beclin 1 & $1: 400$ & $\begin{array}{l}\text { Rabbit monoclonal } \\
\text { (EPR1733Y) }\end{array}$ & Abcam, UK \\
\hline Bcl-2 & $1: 200$ & Mouse monoclonal (3.1) & $\begin{array}{l}\text { Leica BIOSYSTEMS, } \\
\text { UK }\end{array}$ \\
\hline P62 & $1: 600$ & Mouse monoclonal (SQSTM1) & Abcam, UK \\
\hline
\end{tabular}

Table 1: Primary antibodies, clone, dilutions and source.

\section{Semi-quantitative assessment of immunohistochemical staining}

Expression status was evaluated by scoring both intensity and proportion of tumour cells stained for Beclin $1, \mathrm{Bcl}-2$ and $\mathrm{p}^{62}$. The intensity of the staining was scored as 0 (no staining), 1+ (mild staining), $2+$ (moderate staining) or $3+$ (strong staining). The positive tumour cells were recorded after examining the entire tissue section at $100 \mathrm{X}$ magnification. The proportions of tumour cells stained were scored as follows; 0 for no staining, 1 for $<25 \%$ of tumour stained, 2 for $25-50 \%, 3$ for $51-75 \%$ and 4 for $76-100 \%$.

The sum of the scores for intensity and proportion of tumour stained were taken as the measure of the positivity and described as the following; 0 for negative, 1-2 for low expression, 3-4 for moderate expression and 5-6 for high expression. Considering the distribution of nuclear staining in the tumour cells, positive nuclear expression for p62 was defined as the expression of each protein in $>20 \%$ of tumour cells. To assess for reproducibility, all the slides were blindly rescored and tested for systematic errors using Wilcoxon test.

\section{Results}

This study examined the expression patterns of autophagy related proteins in adenoma and adenocarcinoma of colorectal tissues. H\&E staining was performed on 14 colorectal tissues, which were histologically grouped into colorectal adenoma (7) and colorectal adenocarcinoma, Duke Stage B (7). Immunostaining for Beclin1, Bcl-2 
Citation: Adankwah E, Danquah KO, Gyamfi D, Sampene Ossei PP, Asiamah E, et al. (2018) Nuclear Localisation of Autophagic p62 and Associated Cytoplasmic Beclin-1 and Bcl-2 Expressions in Adenomas and Adenocarcinomas of the Colorectal Regions. J Carcinog Mutagen 9: 314. doi:10.4172/2157-2518.1000314

Page 3 of 6

and p62 was performed and the expression levels, using a Quick Score scale, were quantified.

The levels of expression of Beclin 1, Bcl-2 and p62 in both colorectal adenoma and adenocarcinoma cases are shown (Table 2).

\section{Cytoplasmic expression of Beclin 1 in colorectal adenomas and adenocarcinomas}

Beclin 1 expression pattern in colorectal adenoma and adenocarcinoma was investigated using immunohistochemistry. Of the 7 tubular adenoma cases, 1 case showed a moderate Beclin 1 expression whereas 6 showed a high expression level of Beclin 1 (Table 2 ). There were 3 cases each for moderate and high expression levels in adenocarcinoma of colorectal origin (Table 2). In both adenoma and adenocarcinoma, the Beclin 1 was localised in the cytoplasm (Figure 1). There was no Beclin 1 expression in the control case in which the primary antibody was omitted (Figure 1A).

The normal colonic cells showed moderate cytoplasmic staining at the epithelial layer (Figure 1B) but a strong cytoplasmic expression was observed in the more dysplastic glands of the tubular adenomas (Figure 1C). Additional internal positive control was achieved by the staining of the germinal centre of lymphoid follicle. There was a moderate even distribution of Beclin-1 in tubular adenoma (Figure 1D).

Higher expressions of Beclin 1 were observed in colorectal adenocarcinomas (Figures $1 \mathrm{E}$ and $1 \mathrm{~F}$ ). The average expression levels, as revealed by Quick score scale; indicated tubular adenoma had higher expression level than that of adenocarcinoma.

\begin{tabular}{|l|l|l|}
\hline & \multicolumn{2}{|l|}{ Tumour phenotype, number } \\
\hline Protein Expression & Tubular Adenoma & Duke B Adenocarcinoma \\
\hline Cytoplasmic Beclin 1 & & \\
Negative & 0 & 1 \\
Low & 0 & 0 \\
Moderate & 1 & 3 \\
High & 6 & 3 \\
Total & 7 & 7 \\
\hline Cytoplasmic Bcl-2 & & \\
Negative & 0 & 5 \\
Low & 0 & 2 \\
Moderate & 5 & 0 \\
High & 2 & 0 \\
Total & 7 & 7 \\
\hline Cytoplasmic p62 & & \\
Negative & 0 & 0 \\
Low & 0 & 0 \\
Moderate & 3 & 0 \\
High & 4 & 7 \\
Total & 7 & 7 \\
\hline Nuclear p62 & 7 & \\
Negative & 0 & \\
Positive & & \\
Total & & \\
\hline & & \\
\hline
\end{tabular}

Table 2: Expression patterns of autophagy related proteins in colorectal tumours.

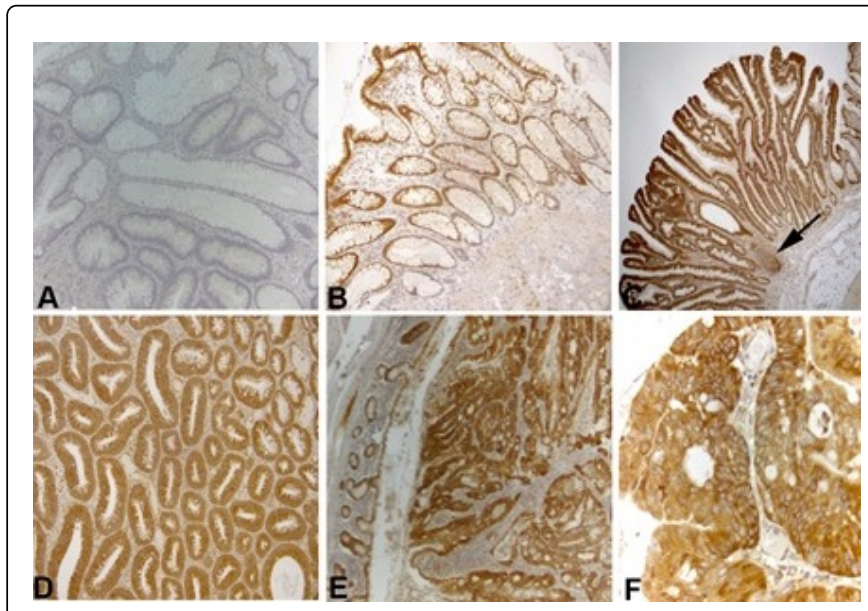

Figure 1: Immunohistochemical patterns of Beclin 1 expression: A) Negative control for Beclin 1, performed with omission of primary antibody. B) Normal colonic mucosa showing moderate cytoplasmic expression of Beclin 1. C) High expression of Beclin 1 in dysplastic glands of tubular adenoma. Insert (arrow), showing the staining of the germinal centre of lymphoid follicle (internal positive control). D) Showing the distribution of moderately stained tubular adenoma. $\mathrm{E}$ and $\mathrm{F}$ ) showing high expression of Beclin 1 in colorectal adenocarcinoma. Magnifications: A, B and D X100, C, E and F X 400.

\section{Cytoplasmic Bcl-2 expression in colorectal cancers}

In order to understand the role of Bcl-2 in the pathogenesis of colorectal tubular adenoma and adenocarcinoma, FFPE sections were immunostained for Bcl-2. Five and two cases, respectively, showed moderate and high $\mathrm{Bcl}-2$ expression levels in adenoma. In contrast, there were 2 cases of adenocarcinoma that showed low expression levels of Bcl-2; and 5 adenocarcinoma cases showed no Bcl-2 expression (Table 2). Negative control was achieved by the omission of the primary antibody (Figure $2 \mathrm{~A}$ ). There was a moderate expression of Bcl-2 at the base of the crypts of normal colonic mucosa cells, adjacent to tumour cells (Figure 2B). Small lymphocytes around the mantle zone of the lymphoid follicles and infiltrating lymphocytes within the tumour stroma were stained intensely for Bcl-2 (Figure 2C). Moderate to high expressions of $\mathrm{Bcl}-2$ were observed in all tubular adenoma cases but for the adenocarcinoma cases, negative to low expressions were observed (Figure $2 \mathrm{E}$ and $2 \mathrm{~F}$ ). The expression of $\mathrm{Bcl}-2$ in both colorectal adenoma and adenocarcinoma was localised in the cytoplasm. As revealed by the Quick Score scale, there was a significant reduction of Bcl-2 expression in colorectal adenocarcinoma than tubular adenoma. 
Citation: Adankwah E, Danquah KO, Gyamfi D, Sampene Ossei PP, Asiamah E, et al. (2018) Nuclear Localisation of Autophagic p62 and Associated Cytoplasmic Beclin-1 and Bcl-2 Expressions in Adenomas and Adenocarcinomas of the Colorectal Regions. J Carcinog Mutagen 9: 314. doi:10.4172/2157-2518.1000314

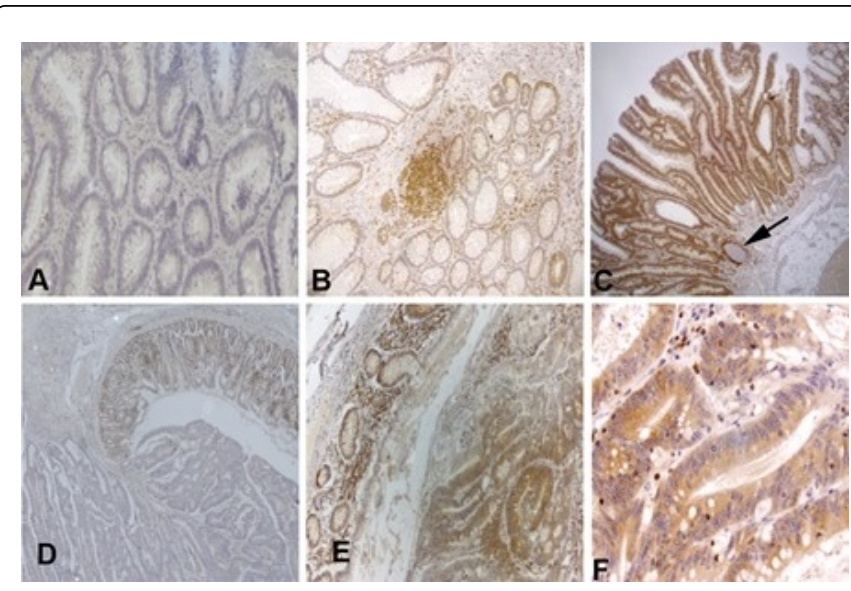

Figure 2: Immunohistochemical patterns of Bcl-2 expression. A) Negative control for Bcl-2, performed with omission of primary antibody. B) Normal colonic tissue showing moderate staining of Bcl-2 at the base of the crypts. C) Moderate cytoplasmic Bcl-2 expression in a tubular adenoma. Insert (arrow), showing intense staining of Bcl-2 by small lymphocytes of the mantle zone of the lymphoid follicle with no staining of the germinal centre. D) Showing no staining for $\mathrm{Bcl}-2$ in a colorectal adenocarcinoma. E and $F$ ) showing weak staining of in a colorectal adenocarcinoma. Magnifications: A, B X100, C, D, E and F X400.

\section{Nuclear and cytoplasmic expression of $p^{62}$ in colorectal cancers}

Several roles are associated with $\mathrm{p}^{62}$ in cancer biology. In this study we examined the localization and expression levels of $\mathrm{p}^{62}$ in colorectal adenoma and adenocarcinoma. All the 7 colorectal adenocarcinoma cases showed a high $\mathrm{p}^{62}$ cytoplasmic expression whilst 3 and 4 adenoma cases showed moderate and high $\mathrm{p}^{62}$ cytoplasmic expression respectively. A negative control was performed by omission of the primary antibody in the staining procedure, and it showed no staining for $\mathrm{p}^{62}$ (Figure $3 \mathrm{~A}$ ). Normal colonic mucosa showed moderate staining of the epithelial cells for $\mathrm{p}^{62}$ (Figure $3 \mathrm{~B}$ ). Moderate $\mathrm{p}^{62}$ expression was observed only in the cytoplasm of tubular adenoma (Figure 3C). Unlike tubular adenoma, which showed cytoplasmic $\mathrm{p}^{62}$ expression, there was p62 expression in both the cytoplasm and the nucleus of the adenocarcinomas cases. A high $\mathrm{p}^{62}$ nuclear expression with moderate $\mathrm{p}^{62}$ cytoplasmic expression was observed in colorectal adenocarcinoma (Figure 3D). There were high $\mathrm{p}^{62}$ cytoplasmic expression and low $\mathrm{p}^{62}$ expression in the nucleus of colorectal adenocarcinoma (Figure $3 \mathrm{E}$ ). In contrast, Figure $3 \mathrm{~F}$ shows moderate to high $\mathrm{p}^{62}$ nuclear expression. In colorectal tubular adenoma, $\mathrm{p}^{62}$ was mainly and exclusively cytoplasmic compared to colorectal adenocarcinoma, in which $\mathrm{p}^{62}$ was localised in both the cytoplasm and the nucleus. The average $\mathrm{p}^{62}$ cytoplasmic expression level in colorectal adenocarcinoma, as indicated in Figure 4, was higher than in tubular adenoma.

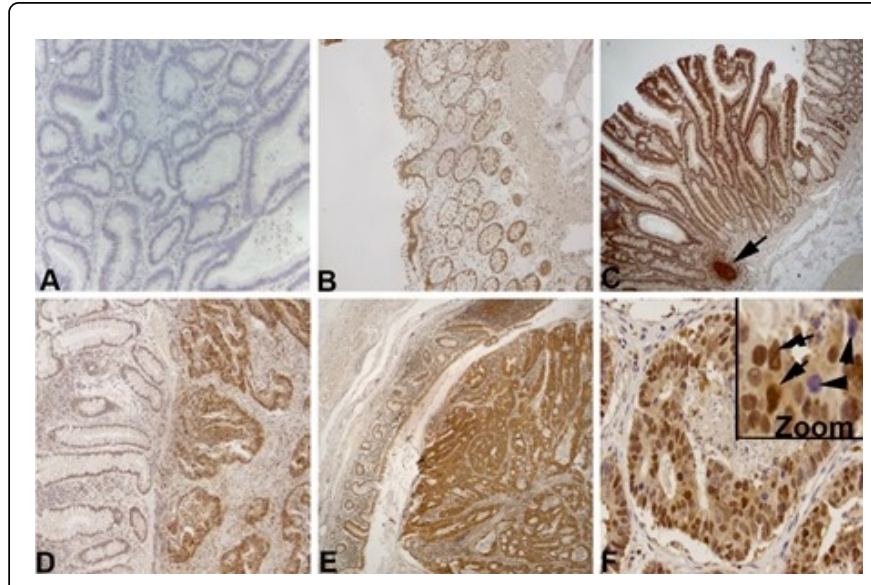

Figure 3: Immunohistochemical patterns of $\mathrm{p}^{62}$ expression. A) Negative control performed with omission of primary antibody. B) Normal colonic mucosa showing moderate cytoplasmic staining of $\mathrm{p}^{62}$ in the epithelium. C) Moderate $\mathrm{p}^{62}$ expression in a tubular adenoma. Insert (arrow) showing intense staining for within the germinal centre of the lymphoid follicle (internal positive control). D) Showing high nuclear $\mathrm{p}^{62}$ expression with moderate cytoplasmic expression as compare to the normal colonic mucosa in a colorectal adenocarcinoma. E) Showing highly intense cytoplasmic $\mathrm{p}^{62}$ expression with low nuclear expression in a colorectal adenocarcinoma. F) Showing moderate to high nuclear $\mathrm{p}^{62}$ expression in a colorectal adenocarcinoma. Magnifications: A, B and D X100, C and E X40, F X400.

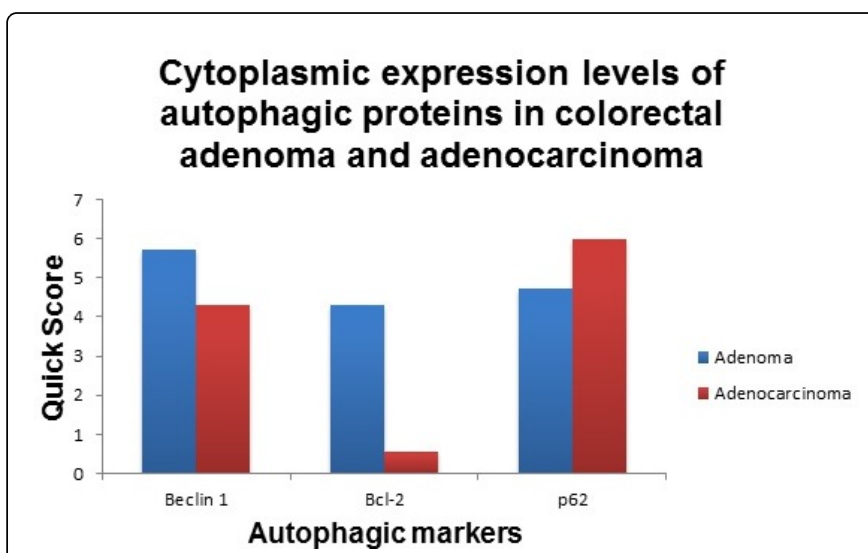

Figure 4: The Cytoplasmic expression levels of autophagic proteins in adenoma and adenocarcinoma of colorectal: Colorectal tissues were immunolabelled with antibodies against Beclin-1, Bcl-2 and $\mathrm{p}^{62}$, followed by secondary antibody staining and development of colour. The intensity of the expression of Beclin-1, Bcl-2 and $\mathrm{p}^{62}$ was semi-quantified in both adenoma and adenocarcinoma.

\section{Discussion}

Colorectal adenomas are lesions that can potentially be transformed to the malignant form, however, it has been estimated that about $95 \%$ of colorectal adenocarcinomas are known to arise from these preexisting lesions [16]. Transformation of normal cells to benign 
adenoma and to malignant colorectal adenocarcinoma involves several cellular processes, including autophagy.

Autophagic processes are essentially involved not only in normal physiological aspect of cells but also in cancer pathogenesis. Studies of several autophagy related biomarkers including Beclin $1, \mathrm{Bcl}-2$ and $\mathrm{p}^{62}$ have shown that deregulation of autophagy may play a role in colorectal adenoma-adenocarcinoma transformation sequence.

In this study, the expression levels of Beclin 1 in normal colon tissue showed a moderate expression of Beclin 1 at the mucosa region. This is consistent with other studies $[6,17]$, indicating that the presence of Beclin 1 as normal component of the normal colonic mucosa and might be involved in initiating basal level of autophagy necessary for cellular homeostasis. Furthermore, we observed that the cytoplasmic Beclin 1 expression ranged from moderate to high expression in tubular adenomas, which was in line with studies by Giatromanolaki et al., where high-grade dysplasia tubular adenomas with or without morphological features of malignant transformation were assessed for Beclin 1 expression. It was speculated that up-regulation of Beclin 1 may occur during the transformation of colorectal adenomas to invasive cancer.

In colorectal adenocarcinomas, Beclin 1 expression levels were from moderate to high. It is considered that Beclin 1 overexpression frequently occurs, but loss or inactivation of Beclin 1 may not occur in tumours of the gastrointestinal tract [4], which is in contrast with the findings [18], where the expression of Beclin 1 is lost in breast and human lung cancer, respectively. However, a low level expression of Beclin 1 was reported in some colorectal cancer subtypes [19]. The possible explanation to these different patterns of expression is that Beclin 1 expression is dependent on the tumour types and can be influenced by other environmental conditions.

Even though we report here of cytoplasmic Beclin 1 expression, others have reported of nuclear localisation of Beclin 1 in some colorectal cancer subtypes [6]. It will be interesting to establish the clinical significance of Beclin 1 localisation in colorectal cancers and its interconnectivity with chemotherapy and prognosis.

The expression pattern of another prominent autophagy protein $\mathrm{Bcl}-2$ was examined in colorectal adenoma and adenocarcinoma. The expression of Bcl-2 was observed to be localised in the epithelial cells at the base of the crypts where the stem cells and the proliferating cells reside, which is consistent with $\mathrm{Han}$ et al on $\mathrm{Bcl}-2$ expression in colorectal cancers. In contrast to the expression of Beclin 1, Bcl-2 expression was absent in most of the adenocarcinoma cases but in only 2 cases that showed low expression. However, in tubular adenomas, Bcl-2 expression was mostly moderate, similar to Goussia et al who reported that higher proportion of adenomas (30.8\%) than carcinomas (16.7\%) expressed Bcl-2. The loss of Bcl-2 expression was also supported by studies from Poincloux et al and Han et al. they reported both negative and low expression for $\mathrm{Bcl}-2$ in colorectal cancers. Considering the moderate expression of $\mathrm{Bcl}-2$ in tubular adenoma cases and the subsequent loss in adenocarcinoma cases, Bcl-2 expression may be an early event in tumour development, confirming the general hypothesis that $\mathrm{Bcl}-2$ might be up-regulated in early stages of the development of colorectal adenomas but is lost in invasive cancer.

A limited number of studies have assessed the intracellular localization of $\mathrm{p}^{62}$ in colorectal tumours. In this study, we observed a moderate cytoplasmic $\mathrm{p}^{62}$ expression pattern in colorectal adenomas, in contrast with a moderate to high nuclear and cytoplasmic expression in colorectal adenocarcinomas. A previous study demonstrated a similar finding of both cytoplasmic and nuclear localisation of $\mathrm{p}^{62}$ but in breast cancers [20]. However, studies by Qian et al. and $\mathrm{Su}$ et al. reported only cytoplasmic expression of p62 in colorectal adenocarcinomas. For the first time, we demonstrate the expression of p62 in both cytoplasmic and nuclear of colorectal adenocarcinomas. Since p62 overexpression has been linked with poor prognosis in breast and human lung cancer [20,21]. It may be postulated that nuclear $\mathrm{p}^{62}$ expression signals a poor prognosis of colorectal cancer development. Nonetheless, more research work needs to be done to understand the significance of $\mathrm{p} 62$ role in nucleocytoplasmic transport in cell proliferation, invasion and chemotherapy response in colorectal adenocarcinomas [22].

\section{Conclusion}

Beclin 1 and p62 expression levels were increased in both colorectal tubular adenomas and adenocarcinomas. This pattern of expression suggests an up-regulation of Beclin 1 and p62 may occur in the development of colorectal adenomas to invasive cancer. Additionally, the expression pattern of Bcl-2 was low in adenocarcinomas and moderately expressed in tubular adenomas, suggesting that Bcl-2 might also contribute to adenoma-adenocarcinoma transition sequence. We provide evidence for the first time of the loss of nuclear expression for $\mathrm{p}^{62}$ in colorectal adenomas but increase expression in colorectal adenocarcinomas.

\section{Ethics Approval}

Ethical approval from the Research Ethics sub-Committee of the University of Westminster, in compliance to the Human Tissue Act (HTA) was given for this study.

\section{Consent for Publication}

Archival human tissue samples were assigned anonymous identifiers using SNOMED coding system, thus this manuscript does not contain any personal data of the human histological samples that were used in this study.

\section{Availability of Data and Materials}

All the data (mainly from images of H\&E and immunohistochemical staining) from which this study's conclusion was made have been presented in this manuscript.

\section{Competing Interest}

We confirm that, regarding this paper; there is no conflict of interest among the authors. Further the work is original and has not been accepted for publication nor is concurrently under investigation elsewhere and will not be published elsewhere without the permission of the Editor.

\section{Authors' Contributions}

Ernest Adankwah (EA), Kwabena Owusu Danquah (KOD), Daniel Gyamfi (DG), Paul Poku Sampene Ossei (PPSO), Emmanuel A Asiamah (EAA) Ibrahim A. Alsafari (IAA) and Tony Madgwick (TM). 
Citation: Adankwah E, Danquah KO, Gyamfi D, Sampene Ossei PP, Asiamah E, et al. (2018) Nuclear Localisation of Autophagic p62 and Associated Cytoplasmic Beclin-1 and Bcl-2 Expressions in Adenomas and Adenocarcinomas of the Colorectal Regions. J Carcinog Mutagen 9: 314. doi:10.4172/2157-2518.1000314

Page 6 of 6

\section{Funding}

This project was funded by Kwame Nkrumah University of Science and Technology (KNUST) Capacity Building Scholarship of the University of Westminster. The funding body was not in any way involved in design of the study and collection, data analysis and interpretation and writing of manuscripts.

\section{Acknowledgement}

I will like to express my profound gratitude to Dr Anthony Warford and Pantelina for their help in this study. Also sincere thanks go to the staff of Queen Hospital, Romford.

\section{References}

1. Mehrpour M, Esclatine A, Beau I, Codogno P (2010) Overview of macroautophagy regulation in mammalian cells. Cell Res 20: 748-762.

2. Swampillai AL, Salomoni P, Short SC (2012) The role of autophagy in clinical practice. Clin Oncol 24: 387-395.

3. Di Bartolomeo S, Corazzari M, Nazio F, Oliverio S, Lisi G, et al. (2010) The dynamic interaction of AMBRA1 with the dynein motor complex regulates mammalian autophagy. J Cell Biol 191: 155-168.

4. Ahn CH, Jeong EG, Lee JW, Kim MS, Kim SH, et al. (2007) Expression of beclin-1, an autophagy-related protein, in gastric and colorectal cancers. APMIS 115:1344-1349.

5. Degenhardt K, Mathew R, Beaudoin B, Bray K, Anderson D, et al. (2006) Autophagy promotes tumor cell survival and restricts necrosis, inflammation, and tumorigenesis. Cancer Cell 10: 51-64.

6. Li BX, Li CY, Peng RQ, Wu XJ, Wang HY, et al. (2009) The expression of beclin 1 is associated with favorable prognosis in stage IIIB colon cancers. Autophagy 5: 303-306.

7. Maiuri MC, Criollo A, Tasdemir E, Vicencio JM, Tajeddine N, et al (2007) BH3-only proteins and $\mathrm{BH} 3$ mimetics induce autophagy by competitively disrupting the interaction between Beclin 1 and $\mathrm{Bcl}-2 / \mathrm{Bcl}-$ X (L). Autophagy 3: 374-376.

8. Priault M, Hue E, Marhuenda F, Pilet P, Oliver L, et al. (2010) Differential dependence on Beclin 1 for the regulation of pro-survival autophagy by Bcl-2 and Bcl-xL in HCT116 colorectal cancer cells. PLoS One 5: e8755.

9. Pattingre S, Tassa A, Qu X, Garuti R, Liang XH, et al. (2005) Bcl-2 antiapoptotic proteins inhibit Beclin 1-dependent autophagy. Cell 122 927-939.
10. Poincloux L, Durando X, Seitz JF, Thivat E, Bardou VJ, et al. (2009) Loss of Bcl-2 expression in colon cancer: a prognostic factor for recurrence in stage II colon cancer. Surg Oncol 18: 357-365.

11. Bjorkoy G, Lamark T, Pankiv S, Overvatn A, Brech A, et al. (2009) Monitoring autophagic degradation of p62/SQSTM1 . Methods Enzymol 452: 181-197.

12. Mathew R, Karp CM, Beaudoin B, Vuong N, Chen G, et al. (2009) Autophagy suppresses tumorigenesis through elimination of p62. Cell 137: 1062-1075.

13. Lee HS, Daniels BH, Salas E, Bollen AW, Debnath J, et al. (2012) Clinical utility of LC3 and p62 immunohistochemistry in diagnosis of druginduced autophagic vacuolar myopathies: a case-control study. PLoS One 7: e36221.

14. Puissant A, Fenouille N, Auberger P (2012) When autophagy meets cancer through p62/SQSTM1. Am J Cancer Res 2: 397-413.

15. Babu JR, Geetha T, Wooten MW (2005) Sequestosome 1/p62 shuttles polyubiquitinated tau for proteasomal degradation. J Neurochem 94: 192-203.

16. Giatromanolaki A, Koukourakis MI, Koutsopoulos AV, Harris AL, Gatter KC, et al. (2013) Autophagy and hypoxia in colonic adenomas related to aggressive features. Colorectal Dis 15: e223-230.

17. Guo GF, Jiang WQ, Zhang B, Cai YC, Xu RH, et al. (2011) Autophagyrelated proteins Beclin-1 and LC3 predict cetuximab efficacy in advanced colorectal cancer. World J Gastroenterol 17: 4779-4786.

18. Jiang ZF, Shao LJ, Wang WM, Yan XB, Liu RY (2012) Decreased expression of Beclin-1 and LC3 in human lung cancer. Mol Biol Rep 39: 259-267.

19. Koukourakis MI, Giatromanolaki A, Sivridis E, Pitiakoudis M, Gatter KC, et al. (2010) Beclin 1 over- and underexpression in colorectal cancer: distinct patterns relate to prognosis and tumour hypoxia. Br J Cancer 103: 1209-1214.

20. Choi J, Jung W, Koo JS (2013) Expression of autophagy-related markers beclin-1, light chain $3 \mathrm{~A}$, light chain $3 \mathrm{~B}$ and $\mathrm{p} 62$ according to the molecular subtype of breast cancer. Histopath 62: 275-286.

21. Inoue D, Suzuki T, Mitsuishi Y, Miki Y, Suzuki S, et al. (2012) Accumulation of p62/SQSTM1 is associated with poor prognosis in patients with lung adenocarcinoma. Cancer Sci 103: 760-766.

22. Fukuhara T, Sakaguchi N, Katahira J, Yoneda Y, Ogino K, et al. (2006) Functional analysis of nuclear pore complex protein Nup62/p62 using monoclonal antibodies. Hybridoma 25: 51-59. 